\title{
Estimation of Cell Cycle States of Human Melanoma Cells with Quantitative Phase Imaging and Deep Learning
}

DOI:

10.1109/ISBI45749.2020.9098458

\section{Document Version}

Accepted author manuscript

Link to publication record in Manchester Research Explorer

\section{Citation for published version (APA):}

Henser-brownhill, T., Ju, R. J., Haass, N. K., Stehbens, S. J., Ballestrem, C., \& Cootes, T. F. (2020). Estimation of Cell Cycle States of Human Melanoma Cells with Quantitative Phase Imaging and Deep Learning. In 2020 IEEE 17th International Symposium on Biomedical Imaging (ISBI) (pp. 1617-1621). IEEE. https://doi.org/10.1109//SBI45749.2020.9098458

\section{Published in:}

2020 IEEE 17th International Symposium on Biomedical Imaging (ISBI)

\section{Citing this paper}

Please note that where the full-text provided on Manchester Research Explorer is the Author Accepted Manuscript or Proof version this may differ from the final Published version. If citing, it is advised that you check and use the publisher's definitive version.

\section{General rights}

Copyright and moral rights for the publications made accessible in the Research Explorer are retained by the authors and/or other copyright owners and it is a condition of accessing publications that users recognise and abide by the legal requirements associated with these rights.

\section{Takedown policy}

If you believe that this document breaches copyright please refer to the University of Manchester's Takedown Procedures [http://man.ac.uk/04Y6Bo] or contact uml.scholarlycommunications@manchester.ac.uk providing relevant details, so we can investigate your claim.

\section{OPEN ACCESS}




\title{
ESTIMATION OF CELL CYCLE STATES OF HUMAN MELANOMA CELLS WITH QUANTITATIVE PHASE IMAGING AND DEEP LEARNING
}

\author{
Tristan Henser-Brownhill ${ }^{1,2}$, Robert J. Ju ${ }^{3,4}$, Nikolas K. Haass ${ }^{4}$, Samantha J. Stehbens ${ }^{3,4}$, Christoph \\ Ballestrem $^{2}$, Timothy F. Cootes ${ }^{1}$ \\ ${ }^{1}$ Division of Informatics, Imaging \& Data Sciences, School of Health Sciences, University of \\ Manchester, Manchester, United Kingdom \\ ${ }^{2}$ Wellcome Trust Centre for Cell Matrix Research, School of Biological Sciences, University of \\ Manchester, Manchester, United Kingdom \\ ${ }^{3}$ The University of Queensland Institute for Molecular Bioscience, Brisbane, QLD, Australia. \\ ${ }^{4}$ The University of Queensland Diamantina Institute, Brisbane, QLD, Australia.
}

\begin{abstract}
Visualization and classification of cell cycle stages in live cells requires the introduction of transient or stably expressing fluorescent markers. This is not feasible for all cell types, and can be time consuming to implement. Labelling of living cells also has the potential to perturb normal cellular function. Here we describe a computational strategy to estimate core cell cycle stages without markers by taking advantage of features extracted from informationrich ptychographic time-lapse movies. We show that a deeplearning approach can estimate the cell cycle trajectories of individual human melanoma cells from short 3-frame $(\sim 23$ minute) snapshots, and can identify cell cycle arrest induced by chemotherapeutic agents targeting melanoma driver mutations.
\end{abstract}

Index Terms - cell cycle, deep learning, melanoma, quantitative phase imaging, ptychography

\section{INTRODUCTION}

The cell cycle is a tightly controlled sequential process that ensures near-perfect replication of a cell's genetic blueprint [1]. In healthy cells, disruption to cell cycle regulatory components leads to activation of intrinsic safety protocols that force cell cycle arrest, DNA repair, or apoptosis (programmed cell death) [2]. If cell cycle checkpoints are missed, this can result in abnormal cell divisions, leading to accumulation of harmful mutations, oncogenic cell transformation, and eventually the formation of cancers or other diseases [2]. Comparison of cell cycle progress in different cell types, cells treated with different chemotherapeutic agents, or between healthy and diseased (a)
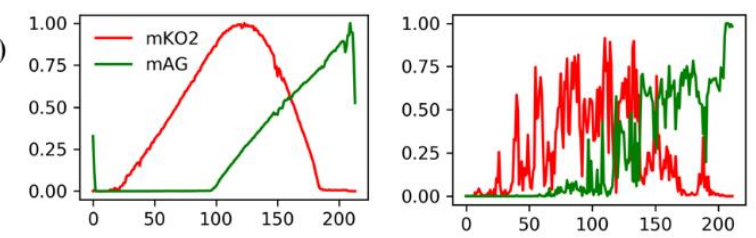

(b)

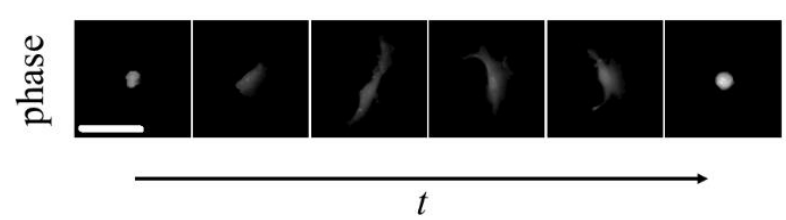

Fig 1. (a) Observed (left) and estimated (right) intensities of a full unseen cell track from the untreated test set (made up of adjacent estimations for individual frames plotted temporally). (b) Visualization of QPI images from the same cell track as above with (6 frames shown, equally spaced along the full track). Scale bar $\approx 75 \mu \mathrm{m}$.

cells, can provide insights into function, treatment effectiveness, or help to identify new drug targets, respectively [2-3]. Typically, the cell cycle is divided into four core stages (Fig. 2a): the growth stage $\left(\mathrm{G}_{1}\right)$ when the cell gathers and synthesizes nutrients required for genomic replication, the DNA synthesis and replication stage (S), a second growth stage $\left(\mathrm{G}_{2}\right)$ where the cell ensures it has all material required for division, and finally mitosis $(\mathrm{M})$ where the cell divides into two new daughter cells [4]. When a cell is not cycling it is said to be in a quiescent $\left(\mathrm{G}_{0}\right)$ state. 
(a)

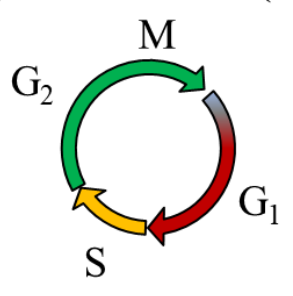

(b)

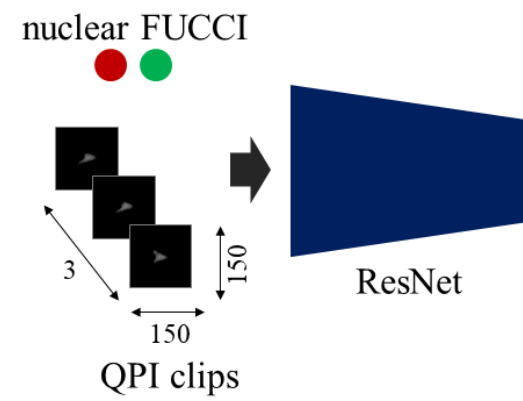

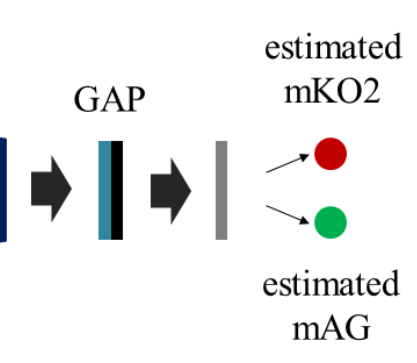

Fig 2. (a) The FUCCI system: during $G_{1}$ of the cell cycle, the mAG tagged fusion protein is actively degraded, whereas the $\mathrm{mKO} 2$ tagged protein is able to express at high levels causing red-orange fluorescence. As the cell enters $\mathrm{S}$ phase, inhibition of the $\mathrm{mAG}$ tagged protein is relaxed, whilst the $\mathrm{mKO} 2$ tagged protein is degraded. During this time the cell expresses both fluorophores at similar levels causing signals to temporarily overlap. As the cell enters $\mathrm{G}_{2} / \mathrm{M}$ only $\mathrm{mAG}$ is observable causing the cell to appear green. (b) Short 3-frame QPI stacks (representing 23 mins) were passed to the residual network, with scaled sum fluorescence intensities for $\mathrm{mKO} 2$ and $\mathrm{mAG}$ associated with the middle frame used as ground truth. The model estimates $\mathrm{mKO} 2$ and $\mathrm{mAG}$ fluorescence corresponding with their nuclear expression levels.

To identify these important stages of the cell cycle in live cells, researchers have, until now, been wholly reliant on fluorescent indicators which are imaged using highpowered microscopy [4]. Such markers are required because cells only show subtle changes in appearance throughout most of the cell cycle, and are mostly transparent using conventional imaging techniques [5]. Unfortunately, the introduction of fluorescent molecules, and their visualization by means of high-powered light emitters, can disrupt normal biological activity and cause cell cycle arrest or death through phototoxic stress [6]. This inevitably makes labelling living cells problematic, and can lead to biased results or failed biological experiments.

Quantitative phase imaging (QPI) techniques are forms of microscopy that can extract detailed information from cells without the need for fluorescent labelling of cellular components or stimulation with high-powered lamps [5]. In this research we utilize ptychography, a form of QPI where a low-energy $650 \mathrm{~nm}$ red laser is moved iteratively over a target region of interest, providing an array of overlapping diffraction patterns that can be used to estimate the phase shift (related to refractive index and thus material density) at each pixel [7].

In this study we used the popular fluorescence ubiquitination cell cycle indicator (FUCCI) system to visualize the cell cycle in a human melanoma cell line [4]. The FUCCI system relies on the introduction of genes that stably express two different fluorescently tagged fusion proteins. For legibility we refer to the fusion proteins by their fluorescent tags: monomeric Kusabiro Orange 2 (mKO2) and monomeric Azami Green (mAG) for the remainder of this paper (see 2. Biological Methods for full details). These express in the cell nucleus at different levels to indicate specific stages of the cell cycle (Fig. $1 \& 2 a$ ). We captured both fluorescence channels, along with correlative ptychographic reconstructions of cells, and used a deep residual convolutional neural network (CNN) to estimate each fluorophore's expression levels based solely on QPI data from single segmented cells. We use the true FUCCI signals as ground truth to train our model, and evaluate its performance on unseen test data representing both typical cell cycle trajectories and $\mathrm{G}_{1}$-arrested cells.

Inspired by the success of recent studies [8-9], we chose to estimate expression levels of $\mathrm{mKO} 2$ and $\mathrm{mAG}$ rather than use this information to indirectly annotate stages (i.e. perform classification of $G_{1}, S, G_{2}$, and $M$ ). This was firstly to avoid subjectivity in judging stage transitions, and risk introducing artificial transition points that do not reflect true cell state; and secondly to assess whether the model might be powerful enough to detect more subtle information about cell cycle state, such as expression of specific proteins. Accordingly, we show that a CNN can estimate markers of cell cycle stage directly from ptychographic images, without using any fluorescent tagging.

\section{BIOLOGICAL METHODS}

The human melanoma cell line WM164 was genotypically characterized [10-11] and grown as described [12]. To generate a stable melanoma cell line expressing the FUCCI constructs, mKO2-hCdt1 (30-120) and mAG-hGem (1-110) [4] were subcloned into a replication-defective, selfinactivating lentiviral expression vector system as described in [13]. The lentivirus was produced by co-transfection of human embryonic kidney $293 \mathrm{~T}$ cells. High-titer viral solutions for mKO2-hCdt1 (30-120) and mAG-hGem (1110) were prepared and used for co-transduction into WM164 cells and subclones were generated by single cell sorting [12,14,15]. Cell line authentication was accomplished by STR fingerprinting (QIMR Berghofer Medical Research Institute, Herston, QLD, Australia). 
Mycoplasma contamination on all cell lines was ruled out via PCR at Garvan Institute. WM164-FUCCI cells were cultured in DMEM (Gibco) supplemented with 10\% FBS (Gibco), $100 \mu \mathrm{g} / \mathrm{ml}$ penicillin streptomycin (Gibco) and $0.1 \mathrm{mM}$ non-essential amino acids. Just prior to imaging cells were treated with $100 \mathrm{nM}$ of the BRAF V600E inhibitor dabrafenib (Selleckchem) and compared with DMSO-treated control cells. These doses were chosen so as to induce $G_{1}$ arrest but low amounts of cell death.

\section{IMAGING AND DATA ACQUISITION}

QPI and correlative fluorescence images were captured every 7.5 minutes over $\sim 60$ hours on a PhaseFocus LiveCyte ptychographic imaging system at $10 \mathrm{X}$ magnification. Imaging regions of interest (ROI) were $750 \times 750 \mu \mathrm{m}$. Fluorophores were excited with a pE-300 ${ }^{\text {white }}$ broadspectrum LED illumination source and were detected using TxRed and FITC Olympus filter cubes. Power and exposures were $25 \%$ for 4 seconds and $35 \%$ for 10 seconds for $\mathrm{mKO} 2$ and $\mathrm{mAG}$, respectively. Individual cells were initially segmented and tracked using the LiveCyte's Cell Analysis Toolbox software (using its proprietary fuzzy cmeans approach), then manually corrected to ensure high quality segmentation and cell tracking throughout the timelapse. Cells crossing the image border were ignored. FUCCI signals for individual cells were obtained from 16-bit fluorescence images in ImageJ by first subtracting background using a sliding paraboloid rolling ball function (50 pixel radius), then adjusting the mean intensity threshold to eliminate remaining background and ensure that only nuclear signal was visible (minimum: 200 for $\mathrm{mKO} 2,500$ for $m A G)$. The sum of pixel intensities inside individual nuclei was then recorded for each cell in each image.

\section{DATA PREPROCESSING}

As fusion protein transfection efficiencies, and therefore general expression levels, likely differ slightly from cell to cell, $\mathrm{mKO} 2$ and $\mathrm{mAG}$ sum nuclear fluorescence signals were scaled between 0 and 1 for each cell, by dividing their recorded intensities by the maximum observed intensity for that cell throughout its entire cell cycle trajectory. Hence, incomplete cell tracks were discarded, and only full cell tracks representing a single completed cell cycle (from $G_{1}$ to M) were used in the final dataset. Individual QPI images of segmented cells were redrawn onto blank backgrounds, and then stacked so that QPI information from the previous, current, and next frames of the time-lapse were available to the model (i.e. sets of 3 consecutive time lapse frames, representing $\sim 23$ minutes each in total). The final dataset contained 30 full cell-tracks, consisting of 5477 3-frame clips, represented as 3-channel 16-bit image stacks (150x150x3), with the FUCCI ground truth associated with the central channel. The mean cell cycle duration was 185 frames with a standard deviation (SD) of 28 frames $(\sim 23 \pm 3$
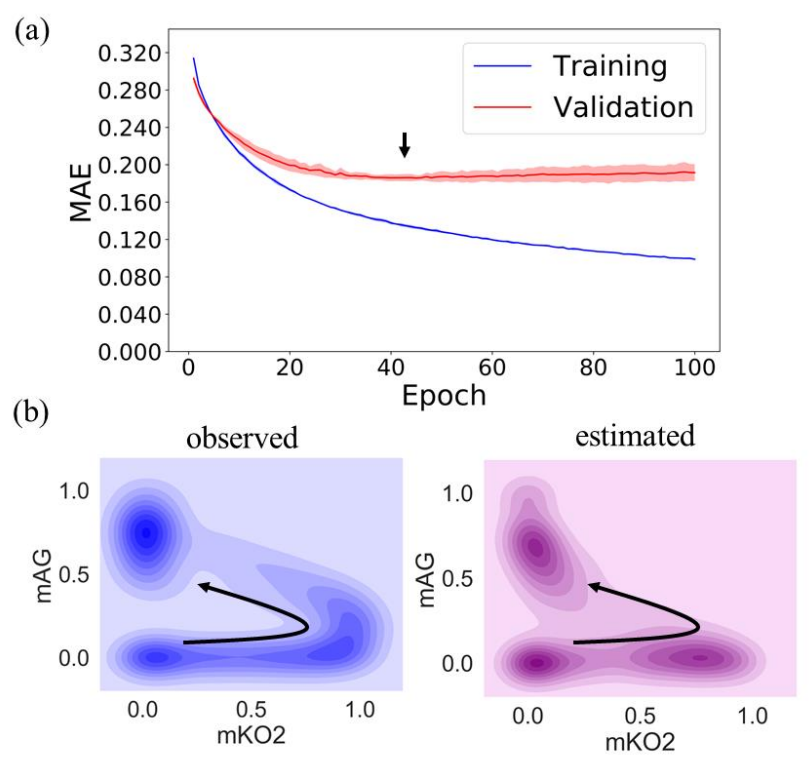

Fig 3. (a) Learning curves showing the median result of 5fold cross-validation, error margins represent 1 median absolute deviation. The thick arrow indicates the epoch were the lowest median loss was obtained, and the early stopping point chosen for final model training. (b) KDEs representing the distributions of $\mathrm{mKO} 2$ and $\mathrm{mAG}$ recorded (left) versus estimated (right) values; arrows indicate direction of typical cell cycle trajectories.

hours). $80 \%$ of the full cell tracks ( 24 cells, 4405 examples) were used for training, whilst 20\% (6 cells, 1072 examples) were held out for testing (i.e. no frames from the same cell track were ever included in both the training and test sets).

\section{DEEP RESIDUAL MODEL}

For this research we used ResNet-50 [16] initiated with weights pre-trained on ImageNet [17] (source: github/fchollet). We added a global average pooling (GAP) layer after the last convolutional layer, followed by 2 neurons activated by sigmoid functions (Fig. 2b). Our resulting model, therefore, outputs 2 separate estimations representing 0-1 scaled $\mathrm{mKO} 2$ and $\mathrm{mAG}$ sum fluorescence levels for each 3-frame QPI clip passed to the model. To avoid overfitting during training we introduced $50 \%$ dropout after the GAP layer. We also performed data augmentation using simple mirroring and rotations to increase the training set size and make our model more robust to changes in orientation. This expanded our training dataset by $16 \mathrm{X}$ to 70480 examples.

We validated the model using 5-fold cross validation and used the median lowest loss as a guide to the number of epochs for training the final model (Fig. 3a). Cross validation folds were split on the list of individual cell tracks, not 3-frame clips, to ensure QPI frames from the 
same cell were never used for both training and validation at the same time. The final model was trained for 45 epochs using stochastic gradient descent with Nesterov momentum of 0.9 and decay of 1e- 6 . We used a learning rate of 1e-5. Our loss function was mean absolute error (MAE). Training was performed using a single Nvidia GeForce GTX 1080 (8GB).

\section{RESULTS}

\subsection{General Performance}

MAE was 0.11 for $\mathrm{mKO}$ 2, and 0.06 for mAG.. Disparities between estimated and ground-truth fluorescence distributions are visualized in Fig. $3 \mathrm{~b}$ using bivariate kernel density estimations (KDE) with bandwidth $b w$ defined by Scott's rule $(\log (b w)=-\log (n) /(d+4))$ where $n$ is the number of points and $d$ the number of dimensions [18].

Correlations between estimated and ground-truth FUCCI confirmed that $\mathrm{mKO} 2$ estimations show a weaker correlation with observed $\mathrm{mKO} 2$ signal (Pearson's r: 0.56) than $\mathrm{mAG}$ estimations with recorded $\mathrm{mAG}(0.70)$; although both cases showed statistically significant correlations with the ground truth $(\mathrm{P}<0.001)$.

\subsection{Performance on $G_{1}$ arrested cells}

Dabrafenib is a small molecule inhibitor of mutated forms of the oncogene BRAF often found in aggressive melanoma [19]. Treatment with dabrafenib causes $\mathrm{G}_{1}$ cell cycle arrest in mutated cells, including the WM164 cell line [15,20,21]. We wanted to observe whether our model could detect this, so we measured FUCCI fluorescence in 3 cell tracks (703 examples) representing melanoma cells blocked with dabrafenib. Cells were tracked following mitosis of a mother cell for at least the mean typical cell cycle duration (see: 3.1). Because mAG would never be expressed at mitotic levels in arrested cells, to generate ground truth we instead scaled the detected $\mathrm{mAG}$ fluorescence by the mitotic mAG observed in the mother cell of each arrested cell studied. Encouragingly our model estimated FUCCI levels corresponding to $\mathrm{G}_{1}$ arrest (Fig. 4) in these cells, with an MAE of 0.12 for $\mathrm{mKO} 2$ and 0.01 for $\mathrm{mAG}$. Linear correlations would not be meaningful for this experiment, given such low variation in ground truth $\mathrm{mKO} 2$ and $\mathrm{mAG}$ fluorescence.

\section{CONCLUSIONS}

We have shown that deep-learning can be used to extract important features from QPI data. Such information enables estimation of the expression levels of cell cycle associated proteins, and detection of cell cycle arrest in cancer cells treated with small molecule therapeutics. Our estimations are currently limited to a single cell line but show great promise. Conducting further work on a large scale, using (a)
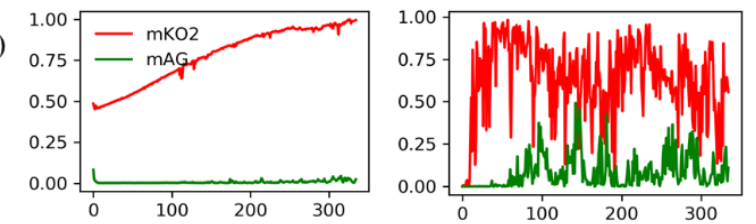

(b)

frame

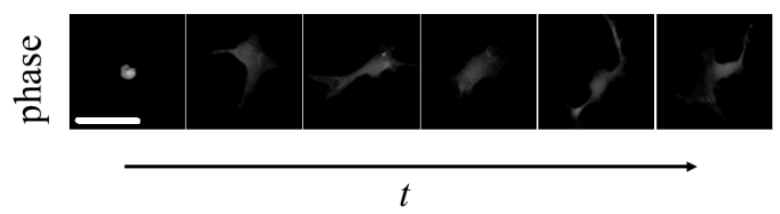

Fig 4. (a) Observed (left) and estimated (right) intensities of a full unseen dabrafenib treated cell track (made up of adjacent estimations for individual frames plotted temporally). (b) Visualization of QPI images from the same cell track as above with (6 frames shown, equally spaced along the full track). Scale bar $\approx 75 \mu \mathrm{m}$.

data from several different cell types, could lead to a generalizable tool for use in the investigation of the cell cycle in a label-free manner. Such a tool would be a powerful platform for evaluating potential new therapeutics.

\section{ACKNOWLEDGMENTS}

This research was supported by a BBSRC doctoral training partnership grant (RCUK project reference: 1924629). We thank both the University of Manchester Bioimaging Core Facility and Phase Focus Ltd. for technical assistance with the LiveCyte QPI imaging system.

\section{REFERENCES}

[1] N. Hustedt, and D. Durocher, "The control of DNA repair by the cell cycle," Nature Cell Biology, vol. 19, no. 1, pp. 1-9, Dec 2017.

[2] K.L. McKinley, and I.M. Cheeseman, "Large-scale analysis of CRISPR/Cas9 cell-cycle knockouts reveals the diversity of p53dependent responses to cell-cycle defects," Developmental Cell, vol. 40 no. 4 pp. $405-420$ Feb 2017.

[3] T. Otto, and P. Sicinski, "Cell cycle proteins as promising targets in cancer therapy," Nature Reviews Cancer, vol. 17, no. 2, pp. 93-115, Jan 2017.

[4] A. Sakaue-Sawano, H. Kurokawa, T. Morimura, et al., "Visualizing spatiotemporal dynamics of multicellular cell-cycle progression," Cell, vol. 132, no. 3, pp. 487-498, Feb 2008. 
[5] R. Kasprowicz, R. Suman, and P. O’Toole, "Characterising live cell behaviour: Traditional label-free and quantitative phase imaging approaches," The International Journal of Biochemistry \& Cell Biology, vol. 84, pp. 89-95, Mar 2017.

[6] S. Wäldchen, J. Lehmann, T. Klein, S. Van De Linde, and M. Sauer, "Light-induced cell damage in live-cell super-resolution microscopy," Scientific Reports, vol. 5, no. 15348, Oct 2015.

[7] J. Marrison, L. Räty, P. Marriott, and P. O'Toole, "Ptychography - a label free, high-contrast imaging technique for live cells using quantitative phase information," Scientific Reports, vol. 3, no. 2369, Aug 2013.

[8] E. M. Christiansen, S.J. Yang, D. M. Ando, A. Javaherian, G. Skibinski, S. Lipnick, E. Mount et al. "In silico labeling: predicting fluorescent labels in unlabeled images." Cell, vol. 173, no. 3, pp. 792-803, Apr 2018.

[9] S.K. Sadanandan, P. Ranefall, S. Le Guyader, and C. Wählby, "Automated training of deep convolutional neural networks for cell segmentation." Scientific Reports, vol. 7, no. 1, pp. 7860, Aug 2017.

[10] K.S. Smalley, R. Contractor, N.K. Haass, A.N. Kulp, G.E. Atilla-Gokcumen, D.S. Williams, et al. "An organometallic protein kinase inhibitor pharmacologically activates p53 and induces apoptosis in human melanoma cells," Cancer Research, vol. 67, no. 1, pp. 209-17, Jan 2007

[11] K.S. Smalley, R. Contractor, N.K. Haass, J.T. Lee, K.L. Nathanson, C.A. Medina, et al. "Ki67 expression levels are a better marker of reduced melanoma growth following MEK inhibitor treatment than phospho-ERK levels," British Journal of Cancer, vol. 96, no. 3. pp. 447-9, Jan 2007.

[12] L. Spoerri, K.A. Beaumont, A. Anfosso, N.K. Haass, "RealTime Cell Cycle Imaging in a 3D Cell Culture Model of Melanoma," in: Z. Koledova (eds) 3D Cell Culture. Methods in Molecular Biology, vol 1612. Humana Press, Jun 2017.

[13] K.S. Smalley, P. Brafford, N.K. Haass, J.M. Brandner, E. Brown, M. Herlyn. "Up-regulated expression of zonula occludens protein-1 in human melanoma associates with $\mathrm{N}$-cadherin and contributes to invasion and adhesion," American Journal of Pathology, vol. 166, no. 5, pp. 1541-54, May 2005.

[14] K.A. Beaumont, A. Anfosso, F. Ahmed, W. Weninger, N.K. Haass, "Imaging- and Flow Cytometry-based Analysis of Cell Position and the Cell Cycle in 3D Melanoma Spheroids". Journal Visualized Experiments, vol. 106, no. e53486, Dec 2015.

[15] N.K. Haass, K.A. Beaumont, D.S. Hill, A. Anfosso, P. Mrass, M.A. Munoz, I. Kinjyo, and W. Weninger, "Real-time cell cycle imaging during melanoma growth, invasion, and drug response," Pigment Cell \& Melanoma Research, vol. 27, no. 5, pp. 764-776, Jun 2014.

[16] K. He, X. Zhang, S. Ren, and J. Sun, "Deep residual learning for image recognition," Proceedings of The IEEE Conference on Computer Vision and Pattern Recognition, pp. 770-778, 2016.
[17] J. Deng, W. Dong, R. Socher, L.J. Li, K. Li, and L. Fei-Fei, "Imagenet: A large-scale hierarchical image database," IEEE Conference on Computer Vision and Pattern Recognition, pp. 248255, Jun 2009.

[18] D. W. Scott, Multivariate Density Estimation: Theory, Practice, and Visualization, 2nd ed., ser. Wiley Series in Probability and Statistics. John Wiley \& Sons, Ltd, Mar 2015.

[19] A. Hauschild, J.J. Grob, L.V. Demidov, T. Jouary, R. Gutzmer, M. Millward, P. Rutkowski, C.U. Blank, W.H. Miller Jr, E. Kaempgen, and S. Martín-Algarra, "Dabrafenib in BRAFmutated metastatic melanoma: a multicentre, open-label, phase 3 randomised controlled trial," The Lancet, vol. 380, no. 9839, pp. 358-365, Jul 2012.

[20] D.R. Menon, S. Das, C. Krepler, A. Vultur, B. Rinner, S. Schauer, K. Kashofer, K. Wagner, G Zhang, E.B. Rad, N.K. Haass, "A stress-induced early innate response causes multidrug tolerance in melanoma," Oncogene, vol. 34, no. 34, pp. 4448-59, Aug 2015.

[21] K.A. Beaumont, D.S. Hill, S.M. Daignault, G.Y. Lui, D.M. Sharp, B. Gabrielli, W. Weninger, N.K. Haass. "Cell cycle phasespecific drug resistance as an escape mechanism of melanoma cells," Journal of Investigative Dermatology, vol. 136, no. 7, pp. 1479-89, Jul 2016. 